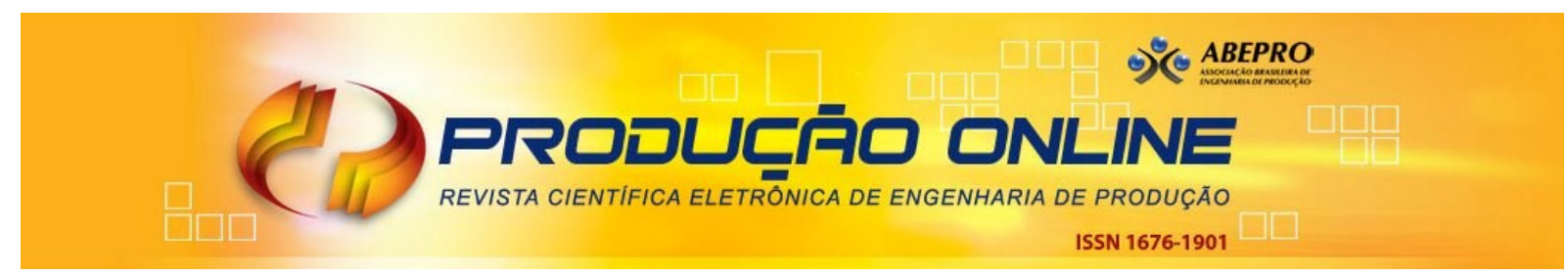

\title{
SUSTENTABILIDADE EM DESENVOLVIMENTO DE PRODUTOS: UMA PROPOSTA PARA A CLASSIFICAÇÃO DE ABORDAGENS
}

\section{SUSTAINABILITY IN PRODUCT DEVELOPMENT: A PROPOSAL FOR CLASSIFICATION OF APPROACHES}

\author{
Patrícia Flores Magnago* E-mail: patriciafm@producao.ufrgs.br \\ João Pedro Ornaghi de Aguiar* E-mail: joaopoa@producao.ufrgs.br \\ Istefani Carisio de Paula* E-mail: istefani@producao.ufrgs.br \\ *Universidade Federal do Rio Grande do Sul, UFRGS, Porto Alegre, RS
}

Resumo: O desenvolvimento de produtos é um importante processo que desencadeia a discussão da sustentabilidade no ambiente empresarial, entretanto qual abordagem empregar? Muitos são os referenciais teóricos, em âmbito acadêmico, que trazem esta perspectiva, como o Capitalismo Natural, o Design for Environment (DfE) e a Análise de Ciclo de Vida (ACV), contudo quais são as diferenças e quando aplicá-las? O objetivo deste estudo foi explorar o entendimento e propor uma classificação, baseada em uma revisão de literatura, para quinze destas abordagens. Os critérios de classificação foram: (i) natureza da abordagem, (ii) nível organizacional relacionado, (iii) aplicação nas macrofases do Processo de Desenvolvimento de Produtos (PDP) e (iv) impacto nas dimensões da sustentabilidade. Também foram realizadas conexões entre elas por meio do levantamento de termos comuns. Este procedimento permitiu concluir que as abordagens, apesar de terem sua origem em áreas de conhecimento distintas, não são mutuamente exclusivas, pelo contrário, cada uma delas se utiliza de conhecimentos anteriores para formar sua base de sustentação. Por fim, é sugerida a aplicação gerencial combinada de abordagens com características complementares.

Palavras-Chave: Abordagens Ambientais. Classificação. Sustentabilidade. Desenvolvimento de Produtos. Gestão.

\begin{abstract}
The product development is a process that addresses sustainability issues inside companies. Many approaches have been discussed in academy concerning sustainability, as Natural Capitalism, Design for Environment (DfE) and Life Cycle Analysis (LCA), but a question arises: which is indicated for what circumstance? This article aim is the proposition of a classification, based on a literature review, for 15 of these approaches. The criteria were: (i) approach nature, (ii) organization level, (iii) integration level in Product Development Process (PDP), and (iv) approach relevance for sustainability dimensions. Common terms allowed the establishment of connections among the approaches. As a result the researchers concluded that, despite they come from distinct knowledge areas they are not mutually excludent, on the contrary, the approaches may be used in a complementary way by managers. The combined use of complementary approaches is finally suggested in the paper.
\end{abstract}

Keywords: Environment. Classification of approach. Sustainability. Product Development. Management.

Revista Produção Online, Florianópolis, SC, v.12, n. 2, p. 351-376, abr./jun. 2012. 


\section{INTRODUÇÃO}

O relacionamento das empresas com a sociedade é realizado fundamentalmente pela oferta de produtos e serviços. Uma parte da responsabilidade pela construção de uma sociedade mais sustentável está associada à capacidade das equipes de Processo de Desenvolvimento de Produtos (PDP) em trazer esta preocupação a todo ciclo de vida dos novos bens e serviços oferecidos aos consumidores. Entre $60 \%$ e $80 \%$ de todos os impactos ambientais causados ao longo da vida de um produto são determinados nas fases iniciais de seu projeto (GRAEDEL; ALLENBY, 1995). Este dado ressalta a importância de que as atividades projetuais dos modelos de PDP devem ser exercidas por colaboradores preocupados em respeitar a capacidade regenerativa da natureza e em desenvolver mecanismos para revisar e reprojetar soluções convencionais (KAWAMOTO et al., 2006).

Uma vez que a relação entre sustentabilidade e PDP está sendo frequentemente discutida no ambiente acadêmico e empresarial, a falta de clareza quanto às categorias das abordagens relacionados ao tema pode gerar incertezas na sua aplicação. A sustentabilidade é um tema de caráter multidisciplinar que nesta pesquisa foi tratado sob o ponto de vista institucional. Esta perspectiva está associada a três objetivos: a responsabilidade ambiental, a responsabilidade social e a geração de valor econômico. Tais objetivos estão de acordo com a visão tridimensional da sustentabilidade de Elkington (1997), que, a partir da tríade planet, people e profit (ambiente, pessoas e lucro), propõe o triple bottom line ou tripé da sustentabilidade. Muitas abordagens vêm nos últimos anos promovendo uma releitura nas técnicas de concepção, projeto e produção industrial de bens, para conter as questões relativas à sustentabilidade (BYGGETH et al., 2007).

Visando contribuir com o aprofundamento no tema, o objetivo deste artigo é a elaboração de uma proposta de classificação para quinze abordagens selecionadas a partir dos referenciais teóricos ligados à sustentabilidade e o PDP. São elas: Desenvolvimento Sustentável, Balanço de Massa, Ecologia Industrial, Ecoeficiência, Capitalismo Natural, Emissão Zero (ZERI), Berço ao Berço, Cadeia de Suprimentos Verde, Análise do Ciclo de Vida (ACV), EcoDesign, Logística Reversa, Produção Mais Limpa, Design for Environment (DfE), QFD for Environment e Emergia. A 
proposição de uma classificação permite a comparação simultânea das abordagens, possibilitando uma visão conjunta. Tal visão conjunta pode contribuir com a seleção da abordagem mais apropriada para os objetivos de uma equipe de PDP inserida em uma empresa de manufatura. Assim, suprindo a lacuna de pesquisa de uso de abordagens sustentáveis integradas aos modelos de desenvolvimento de produtos (AZEVEDO e NOLASCO, 2009; GUELERE FILHO et al., 2009). Para a elaboração da classificação foi levado em consideração os fundamentos que nortearam a proposição de cada uma, por meio de uma revisão teórica. Esta classificação aponta para a identificação de similaridades entre as abordagens, tornando clara a conotação dos conceitos para a academia.

Uma síntese das abordagens é apresentada na seção 2. A partir da avaliação dos referenciais teóricos de cada uma foi possível encontrar dimensões comuns que permitiam a realização de uma comparação contextual. As dimensões converteramse nos critérios de classificação do estudo, que são justificados na seção 3. A realização do detalhamento, definição dos critérios e classificação são apresentadas na seção 4 , em conjunto com a discussão dos resultados encontrados por meio da comparação destes e do levantamento de suas terminologias comuns. Por fim, são apresentadas as conclusões da pesquisa.

\section{SUSTENTABILIDADE E O PDP}

O desenvolvimento de produtos é um processo comum nas empresas de manufatura que invariavelmente impacta o ambiente. Este processo envolve a tradução de informações de mercado em um novo produto disponível para comercialização. Nas fases iniciais, até a formalização de um conceito, o impacto gerado é pequeno. Entretanto, a materialização do conceito e a produção do produto, seu uso e seu descarte, pressupõem a transformação e movimentação de insumos, o que modifica o ambiente (MARGOLIN, 1998; KAZAZIAN, 2005; THACKARA, 2008).

As questões ambientais geralmente não são tratadas em profundidade nos modelos referenciais tradicionais para o PDP, tais como: Rozenburg e Eekels (1995), Crawford e Benedetto (2000), Ulrich e Eppinger (2000), Rozenfeld et al.

Revista Produção Online, Florianópolis, SC, v.12, n. 2, p. 351-376, abr./jun. 2012. 
(2006). Estas questões não recebem devida atenção, possivelmente em razão do grau de complexidade do processo modelado (JACQUES e PAULA, 2007). Novas tendências, trouxeram as questões ambientais explicitamente integradas ao PDP, como as de Abele et al. (2005), Brezet e van Hemel (1997) e a do Desenvolvimento de Produtos Ambientalmente Sustentáveis - DPAS (MAXWELL e VORST, 2003). Estas novas tendências possibilitaram a melhoria de produtos já existentes e a criação de novos produtos reduzindo o impacto no meio ambiente (MORO et al., 2008). Pretende-se que tais produtos apresentem um melhor desempenho ambiental, pois suas funcionalidades, desempenho, estética, qualidade e custo são compatibilizados (NIELSEN e WENZEL, 2001).

Rozenfeld et al. (2006), tratado como modelo referencial neste trabalho, devido sua popularidade na área, divide o PDP em três macrofases: prédesenvolvimento, desenvolvimento e pós-desenvolvimento. Durante a primeira macrofase, a gestão de portfólio e as estratégias empresariais e de produto são tratadas. Na segunda macrofase existe a transformação dos requisitos em características de produtos, prepara-se sua produção e realiza-se o seu lançamento. Por fim, na terceira macrofase, o produto está no mercado, em fase de uso. Ela termina quando a empresa deixa de dar suporte técnico ao produto, descontinuando a produção.

\subsection{Desenvolvimento Sustentável}

Em 1987 é lançado, pelas Nações Unidas, o Relatório Brundtland. Este relatório é conhecido tanto pelo nome da primeira ministra da Noruega na época, Gro Harlem Brundtland, presidenta do evento, quanto pelo título Our Common Future (Nosso Futuro Comum). Este documento é a súmula da Comissão Mundial sobre Ambiente e Desenvolvimento e define o desenvolvimento sustentável.

Desenvolvimento sustentável é o desenvolvimento que atende as necessidades do presente sem comprometer a habilidade das futuras gerações de atenderem as suas próprias necessidades. Ele contém dois elementos basilares: o conceito de necessidade, em especial as necessidades essenciais do mundo menos favorecido, cujo atendimento deve ser priorizado; e a ideia de limitação imposta na habilidade do ambiente em atender as necessidades do presente e do futuro, pelo estado da tecnologia e das organizações sociais (Parte I, Capítulo 2, $1^{\circ}$ parágrafo).

Revista Produção Online, Florianópolis, SC, v.12, n. 2, p. 351-376, abr./jun. 2012. 
Amplamente difundida, essa definição fundamenta grande parte da teoria e da prática compromissada com a sustentabilidade desenvolvida a partir do fim da década de 1980. Ao longo dos anos, a representação visual dessa definição evoluiu de pilares para círculos concêntricos e finalmente círculos que se interseccionam conforme a Figura 1, em uma tentativa de enfatizar o equilíbrio necessário entre as três dimensões da sustentabilidade (IUCN, 2006).

Figura 1 - Evolução das representações da abordagem de desenvolvimento sustentável

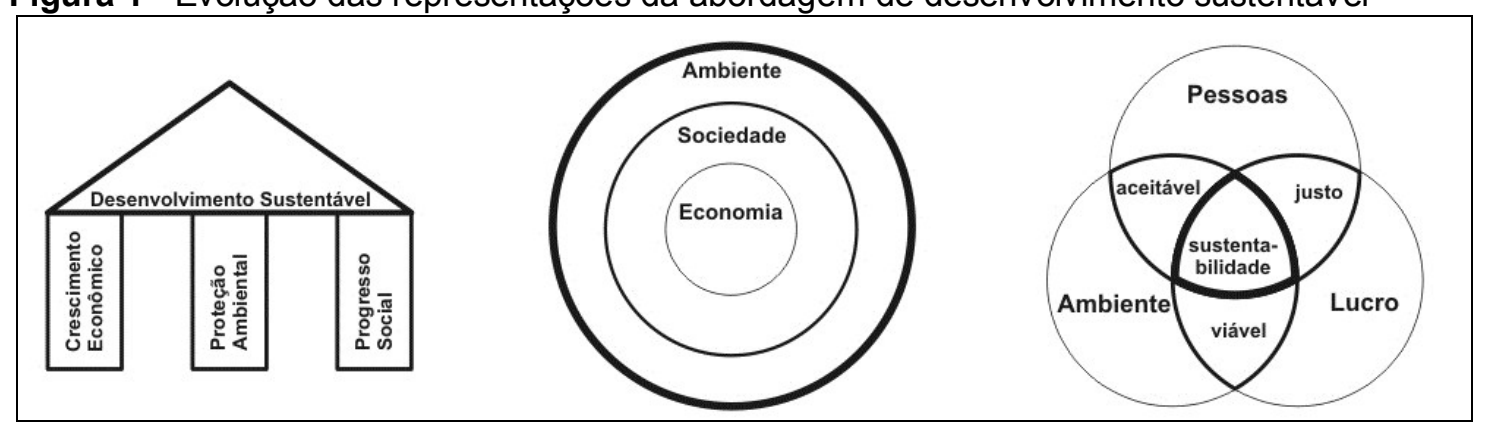

Fonte: adaptado de IUCN (2006)

A definição do termo 'desenvolvimento sustentável' facilita a tarefa de apresentação das distintas abordagens ligadas à sustentabilidade e o PDP conforme se segue.

\subsection{Ecologia Industrial}

A Ecologia Industrial presente em textos desde a década de 1970, propõe um exame integrado das interações entre a indústria e o ambiente. Dessas interações resultam produtos e resíduos. O caminho apontado pela Ecologia Industrial para o desenvolvimento de processos que não desperdicem materiais é a cooperação entre diferentes empresas (GIANNETTI et al., 2003). A Ecologia Industrial trata do entendimento de como o sistema industrial opera, com base no conhecimento do sistema natural dos estudos da Biologia. O objetivo é reestruturar o modo como as indústrias operam a fim de torná-las mais compatíveis com o ambiente.

A ideia da Ecologia Industrial se baseia na analogia com os sistemas naturais. $\mathrm{Na}$ natureza, um sistema ecológico opera em uma rede de conexões, na qual os organismos vivos consomem outros organismos e/ou seus resíduos. A estrutura de um sistema natural e a estrutura de um sistema industrial, ou um sistema econômico, são extremamente semelhantes (FROSH, 1992).

Revista Produção Online, Florianópolis, SC, v.12, n. 2, p. 351-376, abr./jun. 2012. 


\subsection{Ecoeficiência}

Baseado na ideia de 'produzir mais com menos' surge a abordagem Ecoeficiência. O termo foi cunhado pelo Conselho Empresarial Mundial para o Desenvolvimento Sustentável (World Business Council for Sustainable Development - WBCSD) durante a Conferência das Nações Unidas para o Meio Ambiente e o Desenvolvimento (CNUMAD) ou ECO-92, realizada na cidade do Rio de Janeiro. A Ecoeficiência foi apontada como o caminho a ser seguido pelo setor privado para implementar as metas estabelecidas pela Agenda 21. Ela é definida como a entrega de mercadorias e serviços com preços competitivos, que satisfaçam as necessidades humanas, promovam a qualidade de vida enquanto reduzem progressivamente o impacto ambiental até, no mínimo, ao limite de reposição natural dos recursos utilizados (SCHMIDHEINY, 1992).

\subsection{Capitalismo Natural}

O Capitalismo Natural, por sua vez, se desenvolve partindo da premissa da interdepência entre a produção e o uso de objetos manufaturados e a manutenção das fontes naturais dos recursos (HAWKEN et al., 2000). A questão levantada pelos autores é de que forma seria arranjada a economia se ela não estivesse baseada na contabilidade financeira e nos seus conceitos tradicionais. A alternativa proposta seria uma forma de medir o desenvolvimento baseado em indicadores ligados aos recursos naturais. Ainda de acordo com os autores, a próxima etapa da revolução industrial estaria atrelada à conservação de recursos através de processos de produção mais eficientes. A eficiência pode ser alcançada por meio do reuso de materiais, como ocorre nos sistemas naturais; da mudança de valores, partindo da quantidade para o foco em qualidade; e da reparação e manutenção dos recursos da natureza. 


\subsection{ZERI}

Da necessidade de traduzir ideias, princípios e conhecimento científico em projetos concretos é criada, em 1994, por Günter Pauli, a Zero Emission Research and Initiatives (ZERI). ZERI é uma organização internacional, baseada na Universidade das Nações Unidas, no Japão, que busca propor soluções sustentáveis que sejam práticas, viáveis e educativas. É através da emulação dos processos naturais, apoiada no conhecimento científico, que é proposta a criação de produtos, sem a geração de resíduos (PAULI, 1998). Para Pauli, o ZERI serviria como um padrão de eficiência por propor a total eliminação dos resíduos pelo desenvolvimento de cadeias produtivas que agiriam como ecossistemas.

\subsection{Berço ao Berço}

A visão de que resíduos são matéria-prima é o mote para a proposta Berço a Berço, uma oposição à visão tradicional 'berço ao túmulo'. Mais do que uma abordagem, McDonough e Braungart (2002), montaram um sistema de certificação para empresas que redesenham seus processos baseadas na segurança e produtividade regeneradora da natureza, mimetizando o fluxo de nutrientes que ocorre naturalmente utilizando, analogamente, o metabolismo tecnológico.

\subsection{Cadeia de Suprimentos Verde}

A abordagem de Cadeia de Suprimentos Verde trata da aquisição de insumos e equipamentos com o objetivo de melhorar o desempenho tanto financeiro quanto ambiental de uma organização (EPA, 2000). Esse melhor resultado é atingido quando a logística é ajustada para minimizar os impactos ambientais, reduzindo o transporte de produtos tóxicos ou perigosos, muitas vezes com a ajuda da formação de parcerias entre empresas. 


\subsection{Balanço de Massa}

Balanço de Massa é um método quantitativo para mensurar o fluxo de materiais através dos processos de transformação, baseado na lei de conservação de massa de Lavoisier (AYRES e KNEESE, 1969). O balanço de massa é capaz de apontar pontos do processo passíveis de melhoria através de uma detalhada coleta de dados, a elaboração de fluxogramas e o uso de equações próprias para o cálculo do fluxo de materiais.

\subsection{Análise do Ciclo de Vida}

Em 1969, pesquisadores iniciaram, nos Estados Unidos, um estudo interno para a Coca-Cola que serviu de fundamento para o método da Análise do Ciclo de Vida (ACV). O estudo comparou diferentes recipientes para embalagem de bebidas para determinar qual liberaria menos resíduos no ambiente, afetando em menor grau o suprimento de recursos naturais. O estudo foi realizado quantificando-se os materiais e combustíveis utilizados na produção de cada recipiente (EPA, 2006). A ACV é um método quantitativo para a avaliação do impacto ambiental. Segundo Manzini e Vezzoli (2002) essa avaliação é uma operação complexa, pois se deve considerar que o impacto ambiental não é determinado apenas pelo produto ou pelo material que o compõe, mas pelas interações do conjunto de processos envolvidos em todo o seu ciclo de vida. É necessário sistematizar um modelo a partir da extração da matéria-prima até a eliminação final do material. Frente a essa complexidade, muitos softwares específicos para realizar a ACV têm sido desenvolvidos e bancos de dados ligados a órgãos governamentais e universidades foram criados. A norma ISO 14.040 regulamenta as melhores práticas da ACV para certificar empresas que a utilizam (MANZINI; VEZZOLI, 2002).

\subsection{EcoDesign}

O EcoDesign tem sua origem no trabalho de Victor Papanek, originalmente publicado em 1971. Mesmo sem citar diretamente o termo EcoDesign no livro 
Design for the Real World, o autor lança as bases do projeto de produto socialmente justo e ecologicamente responsável. O EcoDesign está contemplado no relatório técnico ISO TR 14.062 de 2002, traduzido na NBR ISO TR 14.062 em 2004, chamada Integração de Aspectos Ambientais no Projeto e Desenvolvimento de Produtos, que leva em conta a função do produto, sua segurança, performance, custo, aceitação no mercado, qualidade, legislação e regulamentos. Para Rozenfeld et al. (2006) a diferença entre EcoDesign e Design for Environment é pouco clara, as duas abordagens compreenderiam o exame sistemático, durante o PDP, dos aspectos relacionadas à proteção ambiental e saúde humana, que devem ser pensados por todos os envolvidos nos projetos.

\subsection{Logística Reversa}

A Logística Reversa busca a reutilização dos produtos através de operações de coleta, desmontagem e reprocessamento de materiais ou peças a fim de assegurar uma estratégia de preços competitivos e engajamento ambiental (DAHLER et al., 2006; CHAVES; BATALHA, 2006; SRIVASTAVA, 2007). A Logística Reversa após o consumo otimiza a reciclagem dos materiais, viabilizando o recondicionamento de componentes, como acontece com as copiadoras da Xerox, que reutilizam peças em produtos novos (MANZINI; VEZZOLI, 2002). Logística reversa de pós-venda trata dos produtos ou de embalagem (SRIVASTAVA, 2007).

\subsection{Produção Mais Limpa}

Segundo o WBCSD (1996), a abordagem Produção Mais Limpa foi introduzida pelo Programa das Nações Unidas para o Ambiente (UNEP) em 1989. Ela é definida pela contínua aplicação de uma estratégia de prevenção ambiental para os processos, produtos e serviços, com o intuito de aumentar a ecoeficiência e reduzir o risco para os seres humanos e para o ambiente. A Produção Mais Limpa requer uma mudança de atitude na gerência das empresas apoiada em políticas ambientais, para a escolha da melhor opção entre as tecnologias disponíveis. 


\subsection{Design for Environment (DfE)}

O EcoDesign e o Design for Environment (DfE) são abordagens próximas, sendo que esta última pertence a uma família de diretrizes de projeto conhecida como Design for $X$ (DfX). DfE foi definido por Fiksel, em 1993, como sendo a consideração sistemática, durante o processo de desenvolvimento de um novo produto, de aspectos projetuais relacionados ao ambiente e com a saúde e segurança do ser humano, que perpassam todo o ciclo de vida do produto. O DfE apresenta diretrizes de projeto ligadas ao fator ecológico, que podem ser usadas como uma lista de verificação pela equipe de desenvolvimento (FIKSEL, 1993).

\subsection{Quality Function Deployment for Environment}

O Japan Environment Management Association for Industry (JEMAI), através do seu comitê de DfE, desenvolveu, em 2001, o Quality Function Deployment for Environment (QFDE ou QFD for Environment) (MASUl et al., 2001). O QFD for Environment é uma ferramenta de auxílio ao projeto para as fases iniciais do desenvolvimento de um produto. Nele, são sugeridos 15 itens chamados 'voz do consumidor ambiental' e 15 'características da qualidade ambiental', que devem ser integradas no desenvolvimento tradicional do Desdobramento da Função Qualidade (QFD). Estes itens adaptam a montagem da matriz da qualidade sob a ótica ambiental, entretanto o objetivo é o mesmo: trazer para o desenvolvimento do produto requisitos do mercado, que algumas vezes estão expressos vagamente, como é o caso dos requisitos ambientais.

\subsection{Emergia}

Partindo da crença de que tudo pode ser medido, inclusive recursos financeiros, em uma unidade de energia (embodied energy ou energy memory), surge uma espécie de contabilidade sócio-ambiental. Ela é a Emergia, uma medida da energia utilizada anteriormente na produção de produtos e serviços, tanto os 
fabricados pelo homem, quanto os feitos pela natureza (ODUM, 1996; ODUM, 1998; ODUM e ODUM, 2003; HAU e BAKSHI, 2004; GIANNETTI, et al. 2006).

Foram apresentadas breves descrições das abordagens ligadas à sustentabilidade e o PDP que são frequentemente mencionadas na literatura. Maiores detalhes sobre estas poderão ser encontrados nos textos originais citados ao longo deste referencial.

\section{PROCEDIMENTOS METODOLÓGICOS}

Para apresentar o escopo deste estudo foi realizada uma adaptação do trabalho de Pedroso e Zwicker (2007) exposta na Figura 2. O escopo envolve as iniciativas das empresas para atingir os propósitos da sustentabilidade, destacando o desenvolvimento de produtos e quinze abordagens representativas de tendências sustentáveis associados a ele. As abordagens selecionadas estão na pauta das discussões atuais da sustentabilidade e o Processo de Desenvolvimento de Produtos (PDP). Na Figura 2, junto a cada iniciativa sustentável, são citados os principais autores ou organizações responsáveis pelo desenvolvimento.

Figura 2 - Escopo do estudo

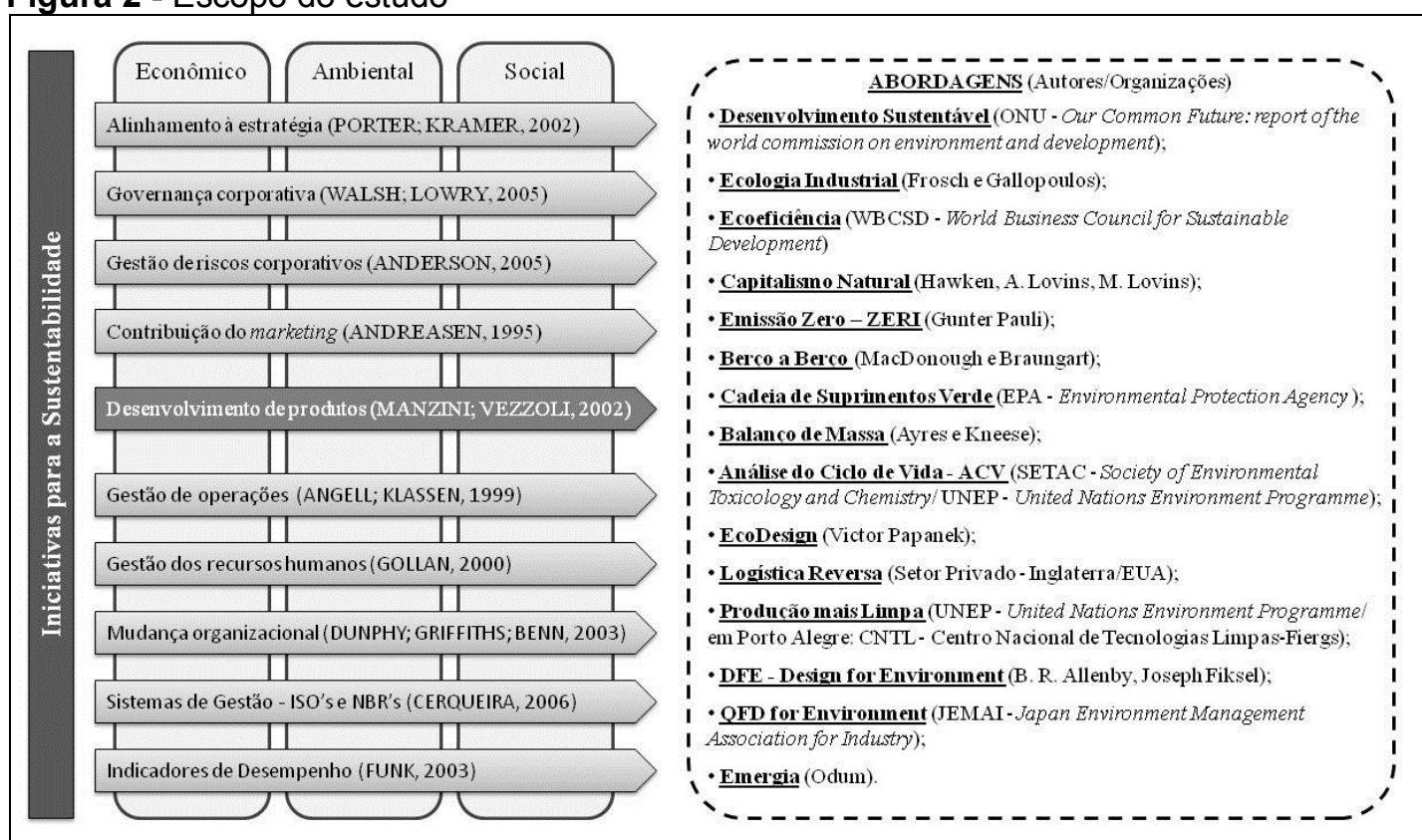

Fonte: Adaptado de Pedroso e Zwicker (2007)

Revista Produção Online, Florianópolis, SC, v.12, n. 2, p. 351-376, abr./jun. 2012. 
Os objetivos desta pesquisa são de caráter exploratório sobre o tema da sustentabilidade e o PDP. Ela está embasada em dados qualitativos e com a classificação dos critérios realizada de forma empírica. O método de trabalho conduzido dividiu-se em cinco etapas: (i) levantamento das abordagens e análise da integração destas ao PDP; (ii) revisão bibliográfica; (iii) desenvolvimento dos critérios de classificação; (iv) classificação; e (v) análise dos resultados.

A classificação foi gerada por meio da interpretação das principais características de cada abordagem acessadas através do referencial teórico. Os livros utilizados nessa pesquisa foram adquiridos, ou consultados na biblioteca da Escola de Engenharia da UFRGS. As principais bases digitais utilizadas para busca de artigos científicos foram: Google Acadêmico (scholar.google.com.br), Scielo (scielo.br), Portal do Conhecimento da Engenharia de Produção da UFRGS (producao.ufrgs.br/publicacoes.asp) e Web of Knowledge (isiknowledge.com). As palavras-chave utilizadas para a busca foram os nomes das abordagens tratadas por este artigo.

A classificação proposta neste artigo foi realizada no segundo semestre do ano de 2009, durante a disciplina 'Tópicos Especiais em Desenvolvimento de Produtos' disponível no Programa de Pós-Graduação em Engenharia de Produção (PPGEP) da Universidade Federal do Rio Grande do Sul (UFRGS). Nove mestrandos do PPGEP com distintas formações (Desenho Industrial, Engenharia de Produção, Engenharia Química, Estatística, Fisioterapia e Medicina), incluindo os autores deste artigo, realizaram o levantamento e debates sobre as práticas e os procedimentos de projeto de produto ligados à sustentabilidade. Foram realizados cerca de doze encontros de três horas e meia, mediante apresentação de artigos em seminários. O resultado dos debates promovidos ajudou a estabelecer os critérios de classificação. A primeira necessidade encontrada foi a de compreensão da natureza de cada abordagem. Isso permitiu definir o quão abrangente ela é. Foi gerado o critério de (i) natureza da abordagem dividido em três níveis paradigma, filosofia e dependente. A segunda necessidade foi a de indicar quais colaboradores de uma dada organização estariam envolvidos com a implementação da abordagem, neste sentido foi criado o critério de (ii) nível organizacional, categorizado também em três níveis: estratégico, tático e operacional. 
A terceira necessidade foi a de indicar, o grau de integração ou vínculo da abordagem com as macrofases do PDP. Para isto, foi desenvolvido o critério de (iii) aplicação nas macrofases do PDP, categorizado em Pré-Desenvolvimento, Desenvolvimento e Pós-Desenvolvimento. A quarta e última necessidade diz respeito a quanto cada abordagem atendia aos princípios sustentáveis, portanto foi instituído o critério de (iv) impacto nas dimensões da sustentabilidade, com as categorias relevância econômica, ambiental e social.

Em paralelo à constituição dos critérios classificatórios, foram identificados na literatura, para cada abordagem, o ano que marca a sua apresentação; a área de conhecimento da qual provém e influência dos autores e organizações que o desenvolveram; e as palavras-chave que estão relacionadas com cada uma. Para o tópico 'palavras-chave' foram selecionadas, dentro da bibliografia, elementos em comum entre as abordagens, gerando uma rede de relações entre as principais fontes dos trabalhos dos autores, e identificação das fontes basilares. A seção a seguir inicia trazendo os resultados encontrados referentes à classificação segundo cada um dos critérios.

\subsection{Resultados e Discussões}

A evolução da preocupação ambiental e do gerenciamento ambiental tem recebido atenção de pesquisadores nas últimas décadas. Duas importantes análises sobre a evolução, respectivamente apresentadas por Beamon (1999) e pelo SENAI.RS (2003) ajudam a entender o contexto sobre os quais as abordagens aqui estudadas foram desenvolvidas. O histórico da preocupação ambiental conta com três períodos. Pode-se dizer que o período de 'disposição' ocorreu até os anos 70, no qual o cenário era de baixa preocupação com o meio ambiente, no qual foram desenvolvidos padrões de qualidade e de emissão de subprodutos. O período denominado de 'tratamento' é subsequente ao de 'disposição' e segue até o inicio dos anos 90. Refere-se ao controle dos resíduos finais (end-of-pipe) com a atitude das empresas ainda ocorrendo de forma isolada ou reativa às normatizações. $O$ período pós 1990, ainda vigente, é o de 'prevenção', no qual se encontram mais frequentemente atitudes empresarias pró-ativas, voluntárias e integradas. 
O gerenciamento ambiental surge a partir do momento em que a preocupação quanto ao tratamento ganha maior força ao longo do tempo. O gerenciamento ambiental também conta com três períodos: Gerenciamento de Riscos, característico da década de 70 que tratou da gestão de resíduos e poluição; Prevenção da Poluição, da década de 80 , que contava com a melhoria de processos para reduzir o volume de materiais; e Gerenciamento do Ciclo de Vida, pós 1990, quando o foco recai sobre o ciclo de vida de produtos e processos através de uma gestão sistêmica. A Figura 3 apresenta uma linha do tempo, com origem em 1950 até o presente ano, apontando a data mais provável de origem das quinze abordagens correspondendo à evolução das questões e gerenciamento ambiental.

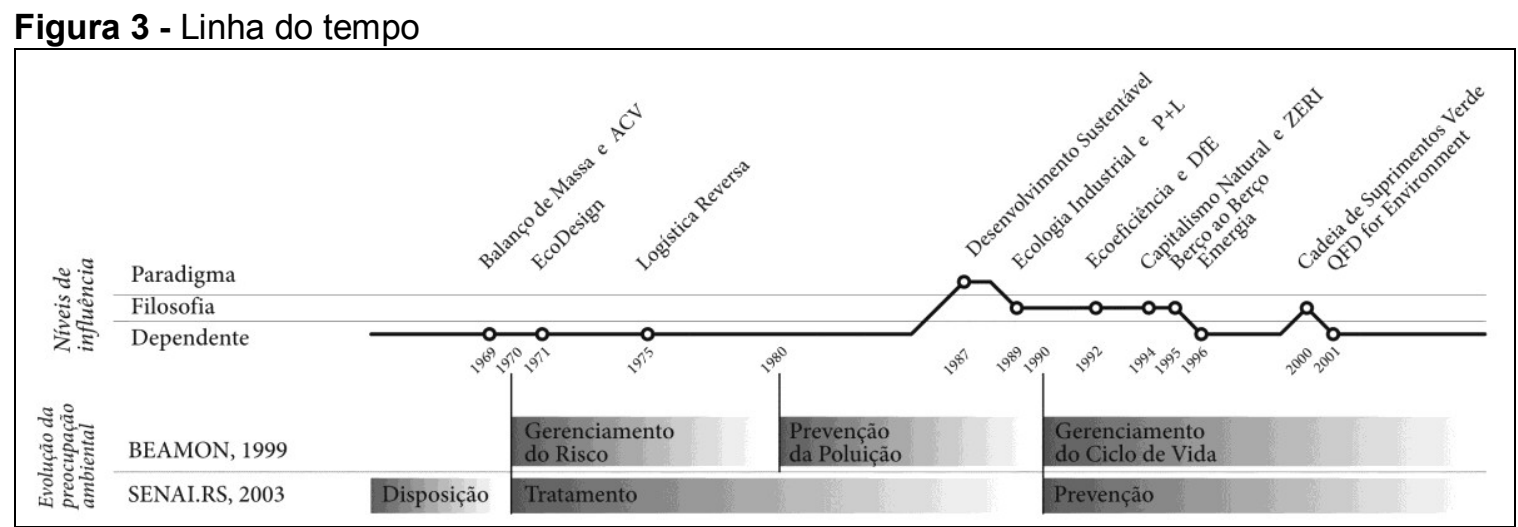

Fonte: Elaborado pelos autores

Observa-se, de uma forma geral, que a preocupação formal com a sustentabilidade é bastante recente quanto período histórico e avançando em concentração de iniciativas. Das primeiras abordagens apresentadas, a ACV e o EcoDesign datados de 1969 e 1971, são os mais referenciados na academia, mas também são aqueles cujas publicações mais divergem quanto às práticas que caracterizariam suas operacionalizações. A segunda mais recente entre as investigadas, a Cadeia de Suprimentos Verde, do ano 2000, exige, mais fortemente que as demais, que exista constante cooperação entre empresas, na forma de parcerias, para garantir o seu sucesso. Assim como acontece com a Cadeia de Suprimentos Verde, que não se sustenta com iniciativas isoladas, a cooperação pode ser uma tendência para as novas abordagens que venham a surgir.

A primeira categoria em que foram classificadas as abordagens diz respeito á sua natureza representada também na Figura 3. A definição do termo 
Desenvolvimento Sustentável, de 1987, foi classificada como paradigma, visto que sugere uma mudança revolucionária na forma como se pensa, se acredita e se faz em relação ao desenvolvimento econômico e industrial, a exemplo do que definia Thomas Kuhn. Para Kuhn (2009), paradigmáticas são as realizações científicas que geram modelos que, por período mais ou menos longo e de modo mais ou menos explícito, orientam o desenvolvimento posterior das pesquisas exclusivamente na busca da solução para os problemas por elas suscitados. Apesar de ser mais uma declaração política do que uma constatação científica, o conceito Desenvolvimento Sustentável se difunde entre as áreas do conhecimento rapidamente após 1987, tornando-se um marco.

Este trabalho adota a definição de filosofia como sendo a investigação crítica e racional sobre a natureza das coisas e as relações entre si. A filosofia geraria um conjunto de princípios para orientação da vida prática (HOUAISS, 2007). Foram classificadas nessa subcategoria as seguintes abordagens por conterem um conteúdo filosófico norteador: Ecologia Industrial, Ecoeficiência, Capitalismo Natural, ZERI, Berço ao Berço e Cadeia de Suprimentos Verde.

A terceira subcategoria para a natureza da abordagem foi chamada de dependente. Isso se deve ao fato de estas variarem entre métodos, procedimentos, técnicas e ferramentas, conforme o uso dado por uma equipe de projetos específica. O contexto modifica a percepção e torna imprecisa uma classificação para este estudo. Logo, são abordagens que dependem da sua aplicação caso a caso para a sua classificação. Oito delas foram classificadas como dependentes: Balanço de Massa, ACV, EcoDesign, Logística Reversa, Produção Mais Limpa, DfE, QFDE e Emergia.

Observando a classificação quanto à área de conhecimento de origem, Figura 4, é possível ver um predomínio ligado a Engenharia e a Administração. Entretanto, é interessante ressaltar a diversidade de áreas envolvidas, o que pode resultar em encontros interdisciplinares de discussão sobre como operacionalizar a sustentabilidade. A interação entre profissionais das ciências naturais com outros das ciências exatas, por exemplo, pode gerar pontos de atrito, e certamente é um dos desafios que as equipes de projeto e seus gestores encontram. 
Figura 4 - Áreas de origem

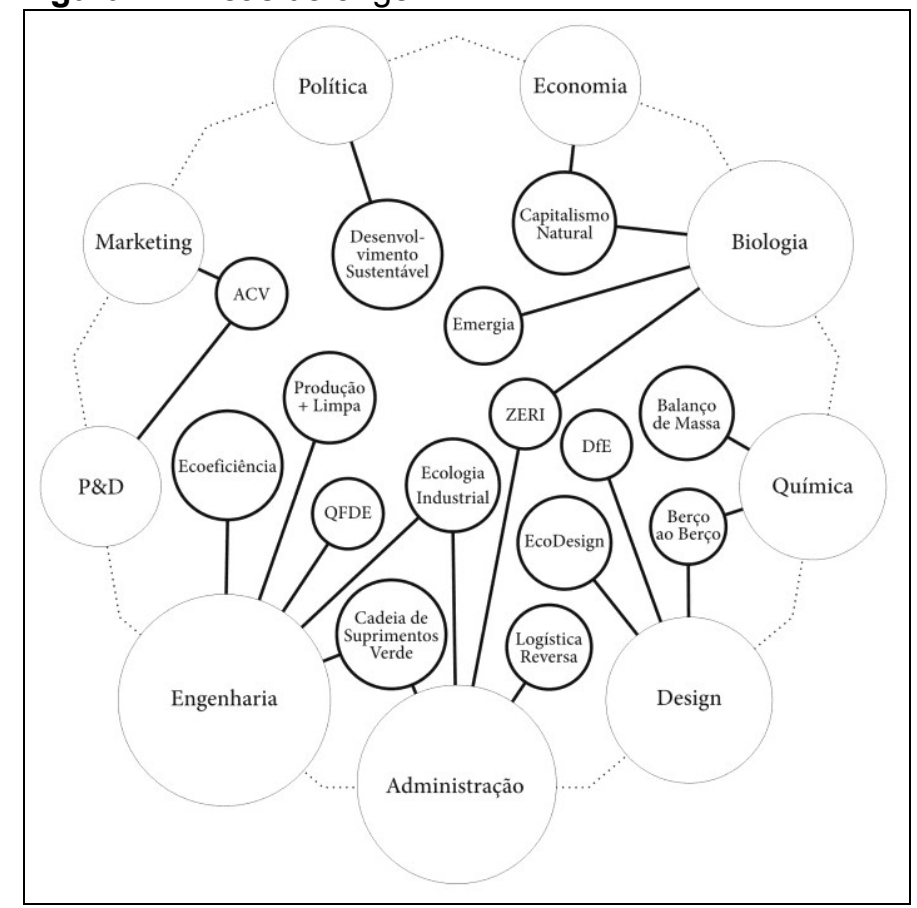

Fonte: Elaborado pelos autores

Foi desenvolvida uma rede de relações através da seleção de palavras-chave comuns. O resultado da rede está na Figura 5. A primeira observação é quanto a não existência de abordagens isoladas, representadas por retângulos na Figura 5. Logo, pontos de convergência entre conhecimentos e vocabulários são encontrados entre essas palavras-chave. A ACV foi a abordagem com mais referências cruzadas, ou seja, sendo a mais citada dentro da literatura das demais. Seus fundamentos, neste sentido, contribuem para os princípios de outras, principalmente as de caráter mais filosóficos, como a Cadeia de Suprimentos Verde e a Ecologia Industrial. Existem entre algumas abordagens fortes relações bidirecionais. Relações bidirecionais, marcadas por setas bidirecionais na Figura 5, são ligações entre suas ideias análogas, inclusive quanto aos seus objetivos, destacam-se três destes relacionamentos: EcoDesign e DfE; DfE e ACV; e Ecoeficiência e Produção Mais Limpa. O EcoDesign e DfE podem ser vistos como os mais similares, sendo, inclusive, tratados como sinônimos por alguns autores.

Entre as palavras-chave, representadas por elipses na Figura 5, a mais comum encontrada na revisão diz respeito à Análise de Input-Output, ou seja, a análise do equilíbrio energético e impacto ambiental, de um sistema ou processo, medindo-se a diferença entre seu inicio (entrada) e fim (saída). Os $3 R$, também se

Revista Produção Online, Florianópolis, SC, v.12, n. 2, p. 351-376, abr./jun. 2012. 
destacam como diretrizes que dinamizaram os problemas de sustentabilidade, são mais conhecidos como o Ciclo 3R (Reduzir, Reutilizar e Reciclar). Pode-se destacar ainda o Biomimetismo, um termo específico alimentado pelas abordagens Capitalismo Natural e ZERI, que promulga o direcionamento de soluções com base naquilo que se pode aprender através da observação da natureza e não no que se pode extrair dela. A biomimética usa um padrão ecológico para inspirar, desta maneira vê e valoriza a natureza de uma forma diferenciada (BENYUS, 1997).

Figura 5- Rede de relações

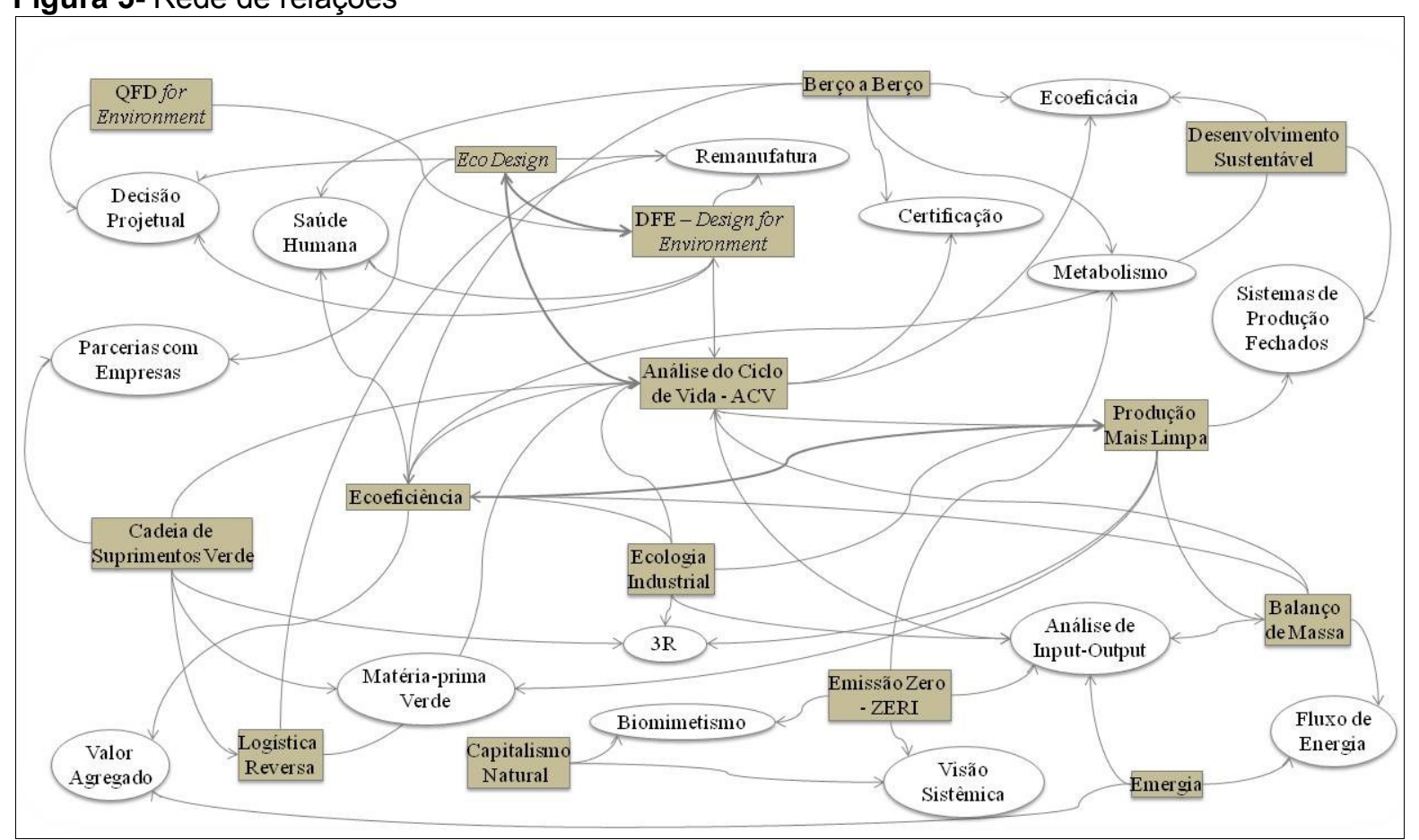

Fonte: elaborado pelos autores

A segunda categoria de classificação foi quanto ao nível organizacional. Ela está dividida em três subcategorias: Estratégico, Tático e Operacional. Se o interesse ou responsabilidade pela implementação da abordagem é da alta gerência ela foi classificada como Estratégica, caso seja do interesse também ao nível dos gestores/supervisores de departamento foi classificada como Tática. Por fim, se a implementação da abordagem recai sobre atividades do nível dos processos ela foi classificada como Operacional. As abordagens apresentam relações com mais de uma subcategoria. Portanto, o grau de relacionamento foi classificada em baixo, médio ou alto. 
Conforme pode ser visto na Figura 6, orientar os processos pela lógica da Ecologia Industrial, por exemplo, se trata de uma decisão estratégica, mas seus princípios não deixam nortear as ações dos demais níveis. Esta abordagem não parece prescrever formas específicas para operacionalizar seus princípios, diferente, por exemplo, das abordagens Berço a Berço ou Produção Mais Limpa que são mais prescritivas do que filosóficas. Neste caso, os colaboradores atuantes nas questões estratégicas podem e devem possuir maior familiaridade com os princípios da Ecologia Industrial, enquanto que os demais colaboradores podem apenas ter uma visão de projetos e rotinas para aplicação das ideias norteadoras desta abordagem. Por outro lado, a ACV é um instrumento característico de controle utilizado pelos gerentes médios, no nível Tático, responsáveis por usar os dados coletados nos demais níveis e dar tratamento a esta informação alimentando tomadas de decisão.

A terceira categoria de classificação é a aplicação nas macrofases do PDP. As macrofases são: (i) Pré-Desenvolvimento, concluída com a aprovação do projeto do produto; (ii) Desenvolvimento, concluída com o lançamento do produto e (iii) PósDesenvolvimento, que se encerra com a retirada do produto do mercado (ROZENFELD et al., 2006). A participação descrição das abordagens nesta categoria também foi definida em alta, média e baixa, como pode ser visto na Figura 6. Enquanto o abrangente Desenvolvimento Sustentável é conceito norteador de todas as fases do PDP, a Logística Reversa e o Balanço de Massa constituem-se em práticas voltadas para as atividades de Desenvolvimento e PósDesenvolvimento, como o projeto, avaliação e recuperação de componentes no final do ciclo de vida do produto.

A quarta categoria de classificação é o impacto da abordagem sobre as dimensões da sustentabilidade. Ela evidencia a ênfase maior da abordagem, sobre uma das dimensões, variando entre Econômico, Ambiental e Social. Os níveis alto, médio e baixo também foram definidos nesta classificação. A Cadeia de Suprimentos Verde e a Produção Mais Limpa podem ser identificadas, segundo a classificação apresentada na Figura 6, com baixo impacto na dimensão Social e forte impacto na dimensão Econômica e Ambiental. Pode-se inferir que este resultado é devido ao contexto de surgimento destas abordagens, em que as questões de sustentabilidade passaram a ser percebidas como geradoras de valor. 
Figura 6 - Classificação das abordagens ligadas à sustentabilidade e PDP

\begin{tabular}{|c|c|c|c|c|c|c|c|c|c|c|c|c|c|c|}
\hline \multirow[b]{2}{*}{ Abordagens } & \multirow[b]{2}{*}{ Descriçăo } & \multirow[b]{2}{*}{ Autor / Organização } & \multirow[b]{2}{*}{ Ano } & \multicolumn{3}{|c|}{$\begin{array}{c}\text { Classificação } \\
\text { quanto a } \\
\text { natureza } \\
\text { da abordagem }\end{array}$} & \multicolumn{2}{|c|}{\begin{tabular}{|c} 
Nivel \\
organizacional
\end{tabular}} & \multicolumn{3}{|c|}{$\begin{array}{c}\text { Aplicacaào } \\
\text { nas macrofases } \\
\text { do PDP }\end{array}$} & \multicolumn{2}{|c|}{$\begin{array}{c}\text { Impacto nas } \\
\text { dimensōes da } \\
\text { sustentabilidade }\end{array}$} & \\
\hline & & & & 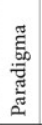 & 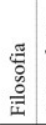 & 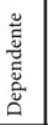 & 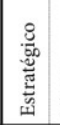 & 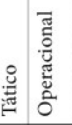 & 焉 & 密 & 总 & 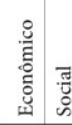 & 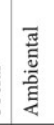 & \\
\hline Balanço de Massa & 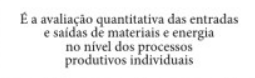 & Ayres $\mathrm{K}$ Knesse & 1969 & & & 0 & & & & & & & & \begin{tabular}{|l} 
Alto \\
Médio \\
Baixo
\end{tabular} \\
\hline $\begin{array}{c}\text { Análise de Ciclo de Vida } \\
\text { ACV }\end{array}$ & 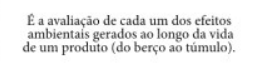 & $\begin{array}{l}\text { SETAC } \\
\text { (Society of Environ Toxic. and Chemistry) } \\
\text { (United Nations Envirer } \\
\text { UNirnment Programme) }\end{array}$ & 1969 & & & 0 & & $\sigma$ & & & & & $a$ & \begin{tabular}{|l} 
Alto \\
Médio \\
Baixo \\
\end{tabular} \\
\hline EcoDesign & 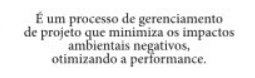 & Victor Papanck & 1971 & & & 0 & - & & $=$ & & & & o- & \begin{tabular}{|l} 
Alto \\
Médio \\
Baixo
\end{tabular} \\
\hline Logística Reversa & 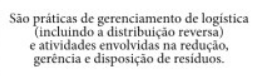 & Setor Privado (Inglaterra/EUA) & 1975 & & & 0 & & & & & & & & \begin{tabular}{|l} 
Alto \\
Médio \\
Baixo
\end{tabular} \\
\hline $\begin{array}{l}\text { Desenvolvimento } \\
\text { Sustentável }\end{array}$ & 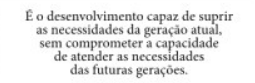 & 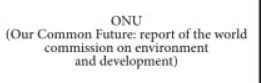 & 1987 & 0 & & & & & $-\infty$ & & $-\infty$ & & $-\infty$ & \begin{tabular}{|l} 
Alto \\
Médio \\
Baixo
\end{tabular} \\
\hline Ecologia Industrial & 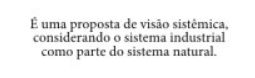 & Frosche Gallopoulos & 1989 & & 0 & & $-a$ & & 二 & & & & 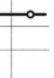 & \begin{tabular}{|l} 
Alto \\
Médio \\
Baixo
\end{tabular} \\
\hline Produção Mais Limpa & 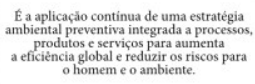 & 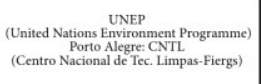 & 1989 & & & 0 & & & $-\infty$ & & & & - & $\begin{array}{l}\text { Alto } \\
\text { Médio } \\
\text { Baixo }\end{array}$ \\
\hline $\begin{array}{l}\text { Design for Environment } \\
\text { DfE }\end{array}$ & 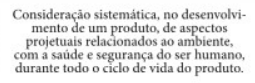 & B. R. Allenby, Joseph Filksel & 1992 & & & 0 & & & 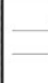 & & - & & & \begin{tabular}{|l} 
Alto \\
Médio \\
Baixo \\
\end{tabular} \\
\hline Ecoeficiência & 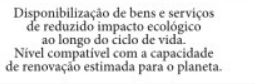 & $\begin{array}{c}\text { WBCDD } \\
\text { World Busincss Council } \\
\text { for Sustainable Development }\end{array}$ & 1992 & & 0 & & & & - & & & & $a$ & \begin{tabular}{|l} 
Alto \\
Médio \\
Baixo
\end{tabular} \\
\hline Capitalismo Natural & 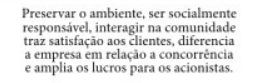 & Hawken, Lovins e Lovins & 1994 & & 0 & & & & $=$ & & & & - & \begin{tabular}{|l} 
Alto \\
Médio \\
Baixo
\end{tabular} \\
\hline Emissão Zero - ZERI & 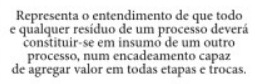 & Gunter Pauli & 1994 & & 0 & & $=a$ & & $=0$ & & & & & \begin{tabular}{|l} 
Alto \\
Médio \\
Baixo
\end{tabular} \\
\hline Berço ao Berço & 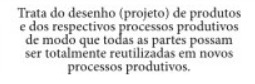 & MacDonough e Braungart & 1995 & & 0 & & & & $-\infty$ & & & & & \begin{tabular}{|l} 
Alto \\
Médio \\
Baixo
\end{tabular} \\
\hline Emergia & 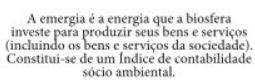 & Howard T. Odum & 1996 & & & 0 & & $-\infty$ & & -0 & & & a & \begin{tabular}{|l} 
Alto \\
Médio \\
Baixo
\end{tabular} \\
\hline $\begin{array}{l}\text { Cadeia de Suprimentos } \\
\text { Verde }\end{array}$ & 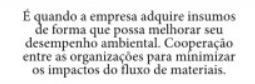 & EPA & 2000 & & 0 & & & & $\rightarrow$ & $-\infty$ & & $=$ & a- & \begin{tabular}{|l} 
Alto \\
Médio \\
Baixo
\end{tabular} \\
\hline QFD for Environment & 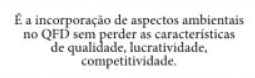 & 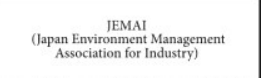 & 2001 & & & 0 & & $-\infty$ & & $-\infty$ & & $-\sigma$ & $\delta_{2}$ & \begin{tabular}{|l} 
Alto \\
Médio \\
Baixo \\
\end{tabular} \\
\hline
\end{tabular}

Fonte: elaborado pelos autores

\section{CONCLUSÃO}

O levantamento bibliográfico de temas relacionados com a sustentabilidade e PDP levou a seleção de quinze abordagens. Uma maior compreensão sobre estes foi alcançada com este trabalho através de uma proposta de classificação. A classificação foi embasada na descrição dos principais objetivos de cada uma e contou com quatro critérios: natureza da abordagem, nível organizacional, aplicação nas macrofases do PDP, impacto da abordagem nas dimensões da sustentabilidade.

Revista Produção Online, Florianópolis, SC, v.12, n. 2, p. 351-376, abr./jun. 2012. 
Os critérios se mostraram adequados para o propósito de análise e foram identificadas duas tendências.

Em um grupo encontram-se as abordagens como o Desenvolvimento Sustentável, o ZERI, a Ecologia Industrial, o Capitalismo Natural, a Ecoeficiência, a Cadeia de Suprimentos Verde e a Produção Mais Limpa, cuja tendência abrangente, estratégica e social se integram melhor às fases iniciais do PDP e ao próprio planejamento estratégico da organização. Este grupo de abordagens estaria relacionado com a formulação de diretrizes norteadoras para as distintas áreas organizacionais, incluindo as equipes de projetos. A adoção destas abordagens têm como pressuposto que os gestores tenham aderido ao novo paradigma do desenvolvimento sustentável. As abordagens possibilitariam mudanças em nível estratégico e não apenas ações pontuais.

No outro grupo encontram-se a Logística Reversa, a Emergia, o Berço ao Berço, o Balanço de Massa, a ACV, o EcoDesign, o DfE e o QFD for Environment. Abordagens com caráter operacional e econômica e que integram melhor às fases finais do PDP. Este grupo de abordagens estaria voltado a equipes de projetos que desejam operacionalizar a sustentabilidade nas práticas de projeto e produção. Sua aplicação é direcionada à melhoria de produtos, processos e serviços específicos. Vale lembrar que a adoção destas ferramentas em nível de desenvolvimento e pósdesenvolvimento deveria estar alinhada com as diretrizes estratégicas da organização. Uma organização que ainda é conduzida pelo paradigma de produção e consumo não sustentável, provavelmente não integralizará qualquer uma destas ferramentas.

As datas de criação e áreas de conhecimento de origem não foram identificadas como características peculiares deste agrupamento. As mais antigas não são necessariamente as mais abrangentes e nem as ligadas à áreas como a Engenharia são menos Estratégicas e mais Operacionais do que as ligadas à áreas como a Administração. Contudo, notou-se que a década de 90 foi a que mais profusa em termos de novas abordagens e as áreas de Engenharia e Administração são, de fato, as mais relevantes.

A elaboração da rede de conexões entre as abordagens por meio de palavras-chave comuns permitiu concluir que estas não são estanques em suas 
práticas, portanto não são mutuamente exclusivas. Pelo contrário, existe uma sinergia e complementaridade de conhecimentos entre elas. No caso do PDP, cada uma contribui de uma maneira particular para reduzir as incertezas inerentes ao processo ao incorporar as questões da sustentabilidade ambiental. Outros estudos também alertaram para a importante questão da adequação da sustentabilidade no PDP, como Finkbeiner et al. (2006). Estes autores afirmam ser necessário esforço substancial para adquirir know-how, informação, experiência e seleção adequadas de diretrizes ambientais que gerem resultados significativos no tempo certo.

Através da classificação apresentada na Figura 6 notou-se que, existe a possibilidade de uso concomitante entre abordagens complementares. Por exemplo, a associação entre a ACV e o Berço ao Berço pode potencializar a atuação da empresa quanto às dimensões na sustentabilidade, tornando-a econômico, social e ambientalmente mais consciente. Já na combinação entre a Ecologia Industrial e a Logística Reversa existe a possibilidade de atingir todos os níveis dentro da organização, estratégico, tático e operacional. Para fomentar o desenvolvimento de produtos ecologicamente corretos pode-se indicar o uso simultâneo do Capitalismo Natural com o EcoDesign. As empresas devem conhecer suas necessidades e capacidades para a adoção de qualquer uma dessas e também a potencialidade da combinação entre elas.

Podem ser identificadas oportunidades de novas investigações de pesquisa oriundas da classificação proposta. Como por exemplo, o levantamento de campo junto a estudantes e especialistas de diferentes áreas de conhecimento verificando o nível de percepção de cada mecanismo sustentável em cada área. Assim como a elaboração de levantamento de campo junto a empresas para a aferição, por meio de uma pesquisa quantitativa, do nível de informações que elas possuem sobre as abordagens e a validação da classificação de cada uma delas quanto aos quatro critérios tratados.

\section{REFERÊNCIAS}

ABELE, E.; ANDERL, R.; BIRKHOFER, H, G. Environmentally-friendly product development: methods and tools. Londres: Springer, p. 0-318, 2005.

ANDERSON, D.R. Corporate survival: the critical importance of sustainability risk management. New York: iUniverse, 2005.

Revista Produção Online, Florianópolis, SC, v.12, n. 2, p. 351-376, abr./jun. 2012. 
ANGELL, L.C.; KLASSEN, R.D. Integrating environmental issues into the mainstream: an agenda for research in operations management. Journal of Operations Management, Amsterdam, v.17, n.5, p.575-598, Aug. 1999.

AYRES, R. U.; KNEESE, A.V. Production, consumption, and externalities. American Economic Review, v. 59, n. 3, p. 282-297, 1969.

AZEVEDO P.S.; NOLASCO, A.M. Requisitos ambientais no processo de desenvolvimento de produtos em indústrias de móveis sob encomenda. In: INTERNATIONAL WORKSHOP. 2, 2009. Anais..., São Paulo, Brazil, 2009.

BEAMON B.M. Designing the green supply chain. Logistics Information Management, v. 12, n. 4, p. 332-342, 1999.

BENYUS, J. M. Biomimética: inovação inspirada pela natureza. São Paulo: Cultrix, 1997.

BREZET, H.; VAN HEMEL, C. Ecodesign: a promising approach to sustainable production and consumption. Paris: UNEP, 1997.

BRUNDTLAND COMMISSION. Our common future: report of the world commission on environment and development. UN, 1987. Disponível em: http://www.undocuments.net/wced-ocf.htm Acesso em: 07/01/10 às 13:59.

BYGGETH, S.; BROMAN, G.; ROBÈRT, K. A Method for sustainable product development based on a modular system of guiding questions. Journal of Cleaner Production, v. 15, p. 1-11, 2007.

CERQUEIRA, J.P. Sistemas de gestão integrados: ISSO 9001, ISSO 14001, OHSAS 18001, SA 8000, NBR16001: conceitos e aplicações. Rio de Janeiro: Qualitymark, 2006.

CHAVES, G. L. D.; BATALHA, M. O. Os consumidores valorizam a coleta de embalagens recicláveis? Um estudo de caso da logística reversa em rede de hipermercados. Gestão e Produção, v.13, n.3, p.423-434, 2006.

CRAWFORD, C.M., BENEDETTO, C.A.D. New product management, EUA: MqcGraw-Hill, International Edition, 2000.

DAHER, C.E.; SILVA, E.P.S.; FONSECA, A.P. Logística reversa: oportunidade para redução de custos através do gerenciamento da cadeia integrada de valor. Brazilian Business Review, v.3, n.1, 2006.

DUNPHY, D.; GRIFFITHS, A.; BENN, S. Organizational change for corporate sustainability, New York: Routledge, 2003.

ELKINGTON, J. Cannibals with forks: the triple bottom line of 21st century business. Oxford: Capstone, 1997.

Revista Produção Online, Florianópolis, SC, v.12, n. 2, p. 351-376, abr./jun. 2012. 
EPA. The lean and green supply chain: a practical guide for materials managers and supply chain managers to reduce cost and improve environmental performance. Environmental Accounting Project, 2000. Disponível em:

http://www.epa.gov/oppt/library/pubs/archive/acct-archive/pubs/lean.pdf Acesso em: $08 / 01 / 10$ às $14: 25$.

EPA. Life cycle assessment: principles and practice. National Risk Management Research Laboratory: Cincinnati , 2006. Disponível em: http://www.epa.gov/nrmrl/lcaccess/pdfs/600r06060.pdf Acesso em: 08/01/10 às 20:31.

FIKSEL, Joseph. Design for environment: an integrated system approach.

Proceedings of the 1993 IEEE International Symposium on Electronics and the Environment. p. 126-131, maio, 1993.

FINKBEINER, M; HOFFMANN, R.; RUHLAND, K.; LIEBHART, D.; STARK, B. application of life cycle assessment for the environmental certificate of the mercedesbenz s-class. International Journal of Life Cycle Assessment, v.11, n.4, p.240246, 2006.

FROSH, Robert A. Industrial ecology: a philosophical introduction. Proceedings of the National Academy of Sciences. USA, 1992.

FUNK, K. Sustainability and performance. Sloan Management Review, Boston, v.44, n.2, p.65-70, Winter, 2003.

GUELERE FILHO, A.; ROZENFELD, H.; PIGOSSO D.C.A. A inserção do design sustentável em um modelo de referência para a gestão do desenvolvimento de produtos. In: SIMPÓSIO PARANAENSE DE DESIGN SUSTENTÁVEL, 1, 2009. Anais..., 2009.

GIANNETTI, B.F.; ALMEIDA, C.M.V.B.; BONILLA, S.H.. Implementação de ecotecnologias rumo à ecologia industrial. RAE-eletrônica, v. 2, n. 1. UNIP: São Paulo, 2003.

GIANNETTI, B.F.; BARBELLA, F.A.; ALMEIDA, C.M.V.B. A combined tool for environmental scientists and decision makers: ternary diagrams and emergy accouting. Journal of cleaner production, p. 201-210, 2006.

GOLLAN, P. Human resources, capabilities and sustainability. In: DUNPHY, D.; BENEVISTE, J.; GRIFFITHS, A.; SUTTON, P. (Eds.). Sustainability: the corporate challenge of the 21st. century. Crows Nest: Allen \& Unwin, p.55-77, 2000.

GRAEDEL, T.E.; ALLENBY, B.R. Industrial ecology. Prentice Hall, 1995.

HAU, J.L. \& BAKSHI, B.R. Promise and problems of emergy analysis. Ecological Modelling, v.178, p. 215-225, 2004.

Revista Produção Online, Florianópolis, SC, v.12, n. 2, p. 351-376, abr./jun. 2012. 
HAWKEN, Paul; LOVINS, Amory; LOVINS, L. Hunter. Natural capitalism: creating the next industrial revolution. New York: Back Bay, 2000.

HOUAISS, Antonio. Dicionário eletrônico da língua portuguesa: versão 2.0. Editora Objetiva: 2007.

IUCN. The future of sustainability: re-thinking environment and development in the twenty-first century. Report of the IUCN renowned thinkers meeting: The World Conservation Union, 2006. Disponível em:

<http://cmsdata.iucn.org/downloads/iucn_future_of_sustanability.pdf>. Acesso em: 8 jan. 2010.

JACQUES, J.J.; PAULA, I.C. Desenvolvimento sustentável de produtos: análise da lacuna entre a prática e filosofia em empresas do rio grande do sul. In: ENCONTRO NACIONAL SOBRE GESTÃO EMPRESARIAL E MEIO AMBIENTE, 9, 2007. Anais .... Curitiba, 2007.

KAZAZIAN, Thierry. Haverá a idade das coisas leves: design e desenvolvimento sustentável. São Paulo: Editora Senac , 2005.

KAWAMOTO, C.I.; SANTOS, F.C.A.; JABBOUR, C.J.C. Combining eco-Design tools in the product development process. In: GLOBAL CONGRESS OF SUSTAINABLE MANUFACTURING, 4, 2006. Anais... São Carlos, SP, 2006.

KUHN, Thomas S. A estrutura das revoluções científicas. 9. ed. São Paulo: Perspectiva, 2009.

MANZINI, E.; VEZZOLI, C. O desenvolvimento de produtos sustentáveis: os requisitos ambientais dos produtos industriais. São Paulo: Editora da Universidade de São Paulo, 2002.

MARGOLIN, Victor. O design e a situação mundial. Rio de Janeiro: UERJ/ESDI, 1998. Arcos - design, cultura, material e visualidade, v. 1.

MASUI, Keijiro; SAKAO, Tomohiko; INABA, Atsushi. Quality function deployment for environment: QFDE (1st report): a methodology in early stage of DfE. In:

PROCEEDINGS OF THE $2^{\text {ND }}$ INTERNATIONAL SYMPOSIUM ON

ENVIRONMENTALLY CONSCIOUS DESIGN AND INVERSE MANUFACTURING (EcoDesign'01). IEEE, 2001.

MAXWELL, D.; VORST, R. van der. Developing sustainable products and services. Journal of cleaner production. v. 11, p. 883-895, 2003.

MCDONOUGH, W.; BRAUNGART, M. Remaking the way we make things: cradle to cradle, New York: North Point Press, 2002.

MORO, J.; MARCON, C.C. ; CORRÊA, J.I. ; ABREU, L.P. Inovação e sustentabilidade de produto: desafios e responsabilidade. In: SIMPÓSIO DE 
ENGENHARIA DE PRODUÇÃO - SIMPEP, 15, 2008. Anais... Bauru, São Paulo., 2008.

NIELSEN, P.H.; WENZEL, H. Integration of environmental aspects in product development: a stepwise procedure based on quantitative life cycle assessment. Journal of Cleaner Production, v.10, p. 247-257, 2002.

ODUM, H.T. Environmental accounting: emergy and environmental decision making. Wiley, New York, 370 p.1996.

ODUM, H.T. Self-organization, transformity, and information. Science, 242, p. 11321139, 1988.

ODUM H.T.; ODUM, B. Concepts and methods of ecological engineering.

Environmental Engineering Science. p. 361, 2003.

PAPANEK, Victor. Design for the real world. Frogmore: Granada, 1980 (1971).

PAULI, G. Upsizing. Como gerar mais renda, criar mais postos de trabalho e eliminar a poluição. Fundação ZERI Brasil: L\&PM, 1998.

PEDROSO, M.C.; ZWICKER, R. Sustentabilidade na cadeia reversa de suprimentos: um estudo de caso do Projeto Plasma. Revista de Administração, São Paulo, v. 42, n. 4, p. 414-430, out./dez. 2007.

PORTER, M.E.; KRAMER, M.R. The competitive advantage of corporate philanthropy. Harvard Business Review, Boston, v.80, n.12, p.56-68, Dec. 2002.

ROZENBURG N.F.M; EEKELS J. Product design: fundamentals and methods, Chichester: John Wiley and Sons, 1995.

ROZENFELD, H.; AMARAL, D.C; FORCELLINI, F.A.; TOLEDO; J.C.; SILVA, S.L.; ALLIPRANDINI, D.H.; SCALICE, R.K.. Gestão do desenvolvimento de produtos: uma referência para a melhoria do processo. Saraiva, São Paulo, 2006.

SCHMIDHEINY, Stephan. Changing course: a global business perspective in development and the environment. Cambridge: MIT Press, 1992.

SENAI.RS. Implementação de programas de produção mais limpa. Porto Alegre, Centro Nacional de Tecnologias Limpas SENAI-RS/UNIDO/INEP, 2003.

SPERANDIO, Sergio A.; DONAIRE, Denis. Produção limpa: da concepção à realidade. SIMPEP. 12, 2005. Anais... Bauru, 07-09 novembro de 2005.

SRIVASTAVA, S. K. Green supply-chain management: a state-of-art literature review. International Journal of Management Reviews, v.9, n.1, p.53-80, 2007. 
THACKARA, John. Plabo B: o design e as alternativas viáveis em um mundo complexo. São Paulo: Saraiva, 2008.

ULRICH, K. T.; EPPINGER, S. D. Product design and devolopment. United States of America: Irwin McGraw -Hill Companies, ed. 2, 2000.

WALSH, M.; LOWRY, J. CSR and corporate governance. In: MULLERAT, R. (Eds.). Corporate social responsibility: the corporate governance of the 21st. century. Hague: Kluwer Law International, p.37-60, 2005.

WBCSD. Eco-efficiency and cleaner production: charting course do sustainability. WBCSD/UNEP, s/d. Disponível em:

<http://www.wbcsd.org/DocRoot/aFQps2TRHhw5tFsl5oZP/eecleanerprod.pdf>. Acesso em: 9 jan. 2010.

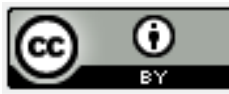

Artigo recebido em 17/09/2010 e aceito para publicação em 23/04/2012. 